7 Sen RP, Walsh TE. Accidental methacholine bronchoprovocation in a laboratory worker. Chest 1991; 99: 1053-1054.

8 Sterk PJ, Fabbri LM, Quanjer PH, et al. Airway Responsiveness: Standardised challenge testing with pharmacological, physical and sensitizing stimuli in adults. Eur Respir J 1993; 6: Suppl. 16, 53-83.

9 Quanjer PH, Stanojevic S, Cole TJ, et al. Multi-ethnic reference values for spirometry for the 3-95 year age range: the global lung function 2012 equations. Eur Respir J 2012; 40: 1324-1343.

10 Miller MR. Does the use of per cent of predicted have any evidence base? Eur Respir J 2015; 45: 322-323.

11 Juniper EF, Syty-Golda M, Harggreave FE. Histamine inhalation tests: inhalation of aerosol via a facemask versus a valve box with mouthpiece. Thorax 1984; 39: 556-557.

12 Ruffin RE, Alpers JH, Cockett AJ, et al. Repeated histamine inhalation tests in asthmatic patients. J Allergy Clin Immunol 1981; 67: 285-289.

\title{
Nasal potential difference of carriers of the W493R ENaC variant with non-cystic fibrosis bronchiectasis
}

\author{
To the Editor:
}

Common underlying conditions leading to bronchiectasis are infection, immune deficiency or cystic fibrosis (CF) [1]. Transgenic mice that overexpress the amiloride-sensitive epithelial sodium channel ENaC mimic the typical features of CF lung disease [2], and correspondingly, it has been proposed that ENaC hyperactivity may predispose to bronchiectasis [3]. The ENaC ion channel consists of three subunits which are encoded by the genes SCNN1A, SCNN1B and SCNN1G. Sequencing of these three genes in 71 subjects with CF-like disease in whom a mutation could not be identified on both CFTR (cystic fibrosis transmembrane conductance regulator) genes uncovered singular cases of disease caused by mutations in SCNN1A [3] and $S C N N B$ [4], and a higher incidence of $E N a C$ polymorphisms [3]. Of nine identified amino acid sequence polymorphisms, one variant in the alpha subunit, named p.W493R-SCNN1A, encoded a hyperactive ENaC channel when tested in a heterologous expression system [3]. Hence AzAD et al. [3] proposed that the p.W493R-SCNN1A ENaC variant could be a global risk factor for the development of lung diseases.

We tested this hypothesis by screening 69 adults with non-CF bronchiectasis from the adult bronchiectasis clinic at Hannover Medical School for the carriage of p.W493R-SCNN1A (rs5742912) (table 1). The study was conducted with the approval of the Ethics Committee of Hannover Medical School (study number: 5391). CF had been excluded by chloride concentrations below the pathological range in the sweat test and/or the absence of any disease-causing mutation in the CFTR coding region in genetic testing. Genomic DNA was amplified with the forward primer 5'-TGCAGGTTAGTGTCCCCTTC and the reverse mismatch primer 5'-CTGGGATGTCACCGATGG by PCR. The 166-bp long PCR product was digested with BseJI (Fermentas; Thermo Fisher Scientific, Vilnius, Lithuania), which cleaves the common W493 allele, and separated by $4 \%$ agarose gel electrophoresis. Six of the 69 subjects with non-CF bronchiectasis were identified as heterozygous carriers of p.W493R-SCNN1A. Thus the incidence of p.W493R-SCNN1A of $8.7 \%$ was significantly higher in the investigated disease cohort than that of $3.1 \%$ in a previously studied healthy control cohort of 739 Mid-European subjects of similar ethnic descent [3] $(p=0.022$, Fisher's exact test). This data demonstrates an association between the carrier status for p.W493R and the manifestation of bronchiectasis.

Next, we wanted to know whether carriage of p.W493R-SCNN1A translates into higher sodium channel activity as indicated by a larger hyperpolarisation response to amiloride in nasal potential difference measurements (NPD) of the upper respiratory epithelium. Five of the six p.W493R carriers were available for an assessment by NPD. NPD measurements were performed according to the Standard Operating Procedure NPD_EU001, version 1.7 (March 2013) of the European Cystic Fibrosis Society Diagnostic Network Working Group, which primarily differs from a publicly available protocol [5] by superfusion of room temperature solutions through a Marquat catheter (model I0202US; Marquat Genie Biomedical, Boissy-Saint-Leger, France). Two subjects presented normal NPD tracings, and two subjects showed a normal response to amiloride, but only a minute response upon exposure to chloride-free solution consistent with the diagnosis of a CFTR-related disorder [6, 7]. However, one subject, a 58-year old female 


\begin{tabular}{|c|c|}
\hline \multicolumn{2}{|l|}{ TABLE 1 Characteristics of the patient cohort ${ }^{\#}$} \\
\hline Subjects $\mathrm{n}$ & 69 \\
\hline Age years & $51 \pm 18$ \\
\hline \multicolumn{2}{|l|}{ Sex } \\
\hline Male & 24 (35) (carriers=3) \\
\hline Female & 45 (65) (carriers=3) \\
\hline FEV $1 \%$ predicted & $47 \pm 27$ \\
\hline Chronic airways colonisation with Pseudomonas aeruginosa & 34 (49) (carriers=3) \\
\hline \multicolumn{2}{|l|}{ Associated conditions? } \\
\hline Idiopathic & $33(48)$ (carriers=2) \\
\hline COPD/emphysema & $8(12)$ \\
\hline Asthma/ABPA & 6 (9) (carriers=1) \\
\hline Post infectious & $5(7)$ \\
\hline Primary ciliary dyskinesia & $5(7)$ \\
\hline Immunodeficiency & 5 (7) (carriers=2) \\
\hline CFTR-related disorder & 3 (4) (carriers=1) \\
\hline Other & $4(6)$ \\
\hline
\end{tabular}

Data are presented as mean \pm SD or $\mathrm{n}(\%)$, unless otherwise stated. $F E V_{1}$ : forced expiratory volume in $1 \mathrm{~s}$; COPD: chronic obstructive pulmonary disease; ABPA: allergic bronchopulmonary aspergillosis; CFTR: cystic fibrosis transmembrane conductance regulator. ${ }^{\#}$ : the number of p.W493R-SCNN1A carriers is listed in brackets; ${ }^{\text {I: }}$ indicates the diagnosis at the beginning of the study.

with non-CF bronchiectasis and underlying asthma complicated by allergic bronchopulmonary aspergillosis, produced a tracing suggestive of a hyperactive ENaC. The basic potential was normal (right nostril $-21 \mathrm{mV}$, left nostril $-27 \mathrm{mV}$ ), but inhibition of the sodium channel caused a large hyperpolarisation to a final value close to $0 \mathrm{mV}(+19 \mathrm{mV},+25 \mathrm{mV})$, which was followed by a similarly large depolarisation upon the exposure to chloride-free gluconate solution $(-21 \mathrm{mV},-21 \mathrm{mV})$. The high sodium conductance caused by the $\mathrm{ENaC}$ channel was balanced by an equivalent chloride conductance mediated mainly by open CFTR channels. Virtually identical NPD profiles have been reported previously for two subjects with CF-like lung disease who were heterozygous carriers for p.W493R-SCNN1A and a mutation in the CFTR gene [3]. Thus there are now three documented cases with bronchiectasis who presented with a large hyperpolarisation upon exposure to amiloride in the NPD.

NPD tracings with normal basic and large hyperpolarisation potentials have been observed in subjects with genetically proven Liddle syndrome which is caused by heterozygous mutation in either the beta or gamma subunit of ENaC [8]. Liddle syndrome is an autosomal dominant disorder characterised by early-onset salt-sensitive hypertension, hypokalaemia and metabolic alkalosis [9]. These clinical features were absent in our index case with non-CF bronchiectasis. The known non-synonymous mutations in the $\mathrm{ENaC}$ subunits cause a broad spectrum of loss-of-function to gain-of-function renal phenotypes, which range from pseudoaldosteronism to Liddle syndrome. According to AzAD et al. [3] and our data a mutant $\mathrm{ENaC}$ may also be occasionally involved in disorders of the lung, including bronchiectasis.

In conclusion, carriage of the p.W493R-SCNN1A polymorphism in the alpha subunit of ENaC has been identified as a risk factor for bronchiectasis, but it is not invariably associated with the phenotype of a hyperactive $\mathrm{ENaC}$ channel in the lung, which promotes the hyper-absorption of sodium ions from the periciliary fluid of the respiratory epithelium and thereby may cause CF-like lung disease. This finding is consistent with the observations that transgenic mice overexpressing SCNN1A do not present with CF-like lung disease [2] and that, of 11 sequence variants identified in the SCNN1A gene of individuals with CF-like lung disease, only the most frequent p.W493R-SCNN1A polymorphism and the singular missense mutation p.F61L-SCNN1A substantially modulate the sodium ion transport capability of the ENaC ion channel in a heterologous test system by more than two-fold [3]. However, factors not covered by the sequencing of the coding region and the exon/intron junctions [3] could also influence $\mathrm{ENaC}$ function, i.e. sequence variants located in deep intronic regions or genetic factors acting in trans that are located elsewhere in the genome. Hence, based on our experience that the most frequent sequence variant in the coding region is not predictive of whether the $\mathrm{ENaC}$ channel will be involved in causing lung disease, in particular bronchiectasis, we would like to recommend that one should start the diagnostic work-up of $\mathrm{ENaC}$ in the context of bronchiectasis of unknown aetiology with NPD measurements followed by genetic testing, if applicable. Based on current knowledge, if there are no other clinical symptoms or implications for $\mathrm{ENaC}$ disease and no indication for $\mathrm{ENaC}$ disease in the NPD measurements, testing of the $S C N N 1 A B G$ genes is not indicated in a clinical setting because of the low clinical consequences for the patient and high costs attached to the extra genetic testing. 
@ERSpublications

ENaC polymorphism p.W493R is associated with bronchiectasis but does not necessarily lead to aberrant ion conductance http://ow.ly/S5Mwj

Jessica Rademacher ${ }^{1}$, Angela Schulz ${ }^{2,3}$, Silke Hedtfeld ${ }^{2}$, Frauke Stanke ${ }^{2,3}$, Felix Ringshausen ${ }^{1,3}$, Tobias Welte ${ }^{1,3}$ and Burkhard Tümmler,3

${ }^{1}$ Dept of Respiratory Medicine, Hannover Medical School, Hannover, Germany. ${ }^{2}$ Clinic for Paediatric Pneumology, Allergology and Neonatology, Hannover Medical School, Hannover, Germany. ${ }^{3}$ Biomedical Research in Endstage and Obstructive Lung Disease (BREATH), German Center for Lung Research, Hannover, Germany.

Correspondence: Burkhard Tümmler, Clinical Research Group, Clinic for Paediatric Pneumology, Allergology and Neonatology, OE 6710, Hannover Medical School, Carl-Neuberg-Str. 1, D-30625 Hannover, Germany.

E-mail: tuemmler.burkhard@mh-hannover.de

Received: July 132015 | Accepted after revision: Aug 122015 | First published online: Oct 92015

Support statement: This work was supported by a grant of the Deutsche Forschungsgemeinschaft (grant number: SFB 621, C7). Funding information for this article has been deposited with FundRef.

Conflict of interest: Disclosures can be found alongside the online version of this article at erj.ersjournals.com

Acknowledgements: We thank Rabea Gatzke (Dept of Respiratory Medicine, Hannover Medical School, Hannover, Germany) for organisational support.

\section{References}

1 McShane PJ, Naureckas ET, Tino G, et al. Non-cystic fibrosis bronchiectasis. Am J Respir Crit Care Med 2013; 188: 647-656.

2 Mall M, Grubb BR, Harkema JR, et al. Increased airway epithelial $\mathrm{Na}^{+}$absorption produces cystic fibrosis-like lung disease in mice. Nat Med 2004; 10: 487-493.

3 Azad AK, Rauh R, Vermeulen F, et al. Mutations in the amiloride-sensitive epithelial sodium channel in patients with cystic fibrosis-like disease. Hum Mutat 2009; 30: 1093-1103.

4 SheridanMB, Fong P, Groman JD, et al. Mutations in the beta-subunit of the epithelial $\mathrm{Na}^{+}$channel in patients with a cystic fibrosis-like syndrome. Hum Mol Genet 2005; 14: 3493-3498.

5 Rowe SM, Clancy JP, Wilschanski M. Nasal potential difference measurements to assess CFTR ion channel activity. Methods Mol Biol 2011; 741: 69-86.

6 Bombieri C, Claustres M, De Boeck K, et al. Recommendations for the classification of diseases as CFTR-related disorders. J Cyst Fibros 2011; 10: Suppl 2, S86-S102.

7 Sermet-Gaudelus I, Girodon E, Sands D, et al. Clinical phenotype and genotype of children with borderline sweat test and abnormal nasal epithelial chloride transport. Am J Respir Crit Care Med 2010; 182: 929-936.

8 Baker E, Jeunemaitre X, Portal AJ, et al. Abnormalities of nasal potential difference measurement in Liddle's syndrome. J Clin Invest 1998; 102: 10-14.

9 Yang K-Q, Xiao Y, Tian T, et al. Molecular genetics of Liddle's syndrome. Clin Chim Acta 2014; 436: 202-206.

\section{Lung positron emission tomography with FDG in patients with haematological malignancies and acute respiratory failure}

To the Editor:

Diagnostic strategy in haematology patients with pulmonary infiltrates relies on different approaches $[1,2]$. Over the past decade, the use of ${ }^{18} \mathrm{~F}$-fluorodeoxyglucose (FDG) positron emission tomography (PET) has been widely used to detect inflammatory and malignant processes, to evaluate the extent of a malignancy, and to ascertain response to therapy. Hот et al. [3] reported diagnostic contribution of FDG-PET in patient with invasive fungal infection. More recently, VERONESI et al. [4] reported PET findings in the diagnostic work-up of screening-detected lung nodules. PET-computed tomography (CT) was highly sensitive for the differential diagnosis of indeterminate nodules detected at baseline, nodules $\geqslant 15 \mathrm{~mm}$ and solid nodules [4]. However, FDG-PET has never been evaluated in unselected patients with acute respiratory failure that complicates haematological malignancies. 